\title{
No evidence of hemoglobin damage by SARS-CoV-2 infection
}

\section{Anthony W. DeMartino,,$^{1^{\star}}$ Jason J. Rose,${ }^{1,2,3^{*}}$ Matthew B. Amdahl, ${ }^{1}$ Matthew R. Dent, ${ }^{1}$ Faraaz A. Shah, ${ }^{2,4.5}$ William Bain, ${ }^{2,4.5}$ Bryan J. McVerry, ${ }^{2,5}$ Georgios D. Kitsios, ${ }^{2,5}$ Jesús Tejero ${ }^{1,2,3,6}$ and Mark T. Gladwin ${ }^{1,2,3}$}

${ }^{1}$ Heart, Lung, Blood, and Vascular Medicine Institute, University of Pittsburgh; ${ }^{2}$ Division of Pulmonary, Allergy and Critical Care Medicine, University of Pittsburgh; ${ }^{3}$ Department of Bioengineering, University of Pittsburgh; ${ }^{4}$ VA Pittsburgh Healthcare System; ${ }^{5}$ The Acute Lung Injury Center of Excellence, University of Pittsburgh and ${ }^{6}$ Department of Pharmacology and Chemical Biology, University of Pittsburgh, Pittsburgh, PA, USA

${ }^{\star} A W D$ and JJR contributed equally as co-first authors

\section{ABSTRACT}

T he severe acute respiratory syndrome coronavirus-2 (SARS-CoV-2) disease (COVID-19) has affected over 22 million patients worldwide as of August 2020. As the medical community seeks better understanding of the underlying pathophysiology of COVID-19, several theories have been proposed. One widely shared theory suggests that SARS-CoV-2 proteins directly interact with human hemoglobin $(\mathrm{Hb})$ and facilitate removal of iron from the heme prosthetic group, leading to the loss of functional hemoglobin and accumulation of iron. Herein, we refute this theory. We compared clinical data from 21 critically ill COVID-19 patients to 21 non-COVID-19 acute respiratory distress syndrome (ARDS) patient controls, generating hemoglobin-oxygen dissociation curves from venous blood gases. This curve generated from the COVID-19 cohort matched the idealized oxygen-hemoglobin dissociation curve well (Pearson correlation $\mathrm{R}^{2}=0.97, P<0.0001$; a coefficient of variation of the root-mean-square deviation $[\mathrm{CV}(\mathrm{RMSD})]=7.3 \%)$. We further analyzed hemoglobin, total bilirubin, lactate dehydrogenase, iron, ferritin, and haptoglobin levels. For all analyzed parameters, patients with COVID-19 had similar levels compared to patients with ARDS without COVID-19. These results indicate that patients with COVID-19 do not exhibit any hemolytic anemia or a shift in the normal hemoglobin-oxygen dissociation curve. We therefore conclude that COVID-19 does not impact oxygen delivery through a mechanism involving red cell hemolysis and subsequent removal of iron from the heme prosthetic group in hemoglobin.

\section{Introduction}

The severe acute respiratory syndrome coronavirus-2 (SARS-CoV-2) pandemic has progressed around the globe with over 22 million cases at the time of this writing. In the early stages of the SARS-CoV-2 disease (COVID-19) pandemic, there were reports of unique clinical phenotypes in affected patients. One study of the initial experiences of critically ill patients in Italy described an atypical form of viral pneumonia-induced acute respiratory distress syndrome (ARDS) with normal lung compliance and low ventilation-to-perfusion ratio. ${ }^{1}$ Initial reports had suggested very high mortality rate $(>80 \%)^{2}$ for patients with respiratory failure from SARSCoV-2 disease (COVID-19), compared to pre-COVID-19 ARDS mortality rates in the range of 30-40\%. ${ }^{3}$ A clinical syndrome of "silent" or "happy" hypoxemia has been widely observed, with patients exhibiting minimal dyspnea or signs of neurocognitive dysfunction despite severe hypoxemia detected via pulse oximetry. ${ }^{4} \mathrm{~A}$ number of hospitalized COVID-19 patients appear to have significant hemostatic activation, with $25-31 \%$ prevalence of venous thromboembolism observed in some cohorts. ${ }^{5,6}$ More recent clinical studies have shown that the mechanical ventilation requirements in COVID-19 patients are similar to populations of patients enrolled in ARDS clinical trials without COVID-19.,8 Further observational studies
Ferrata Storti Foundation
Haematologica 2020
Volume 105(12):2769-2773

\section{Correspondence:}

JASON J. ROSE

rosejj@upmc.edu

Received: June 24, 2020.

Accepted: August 27, 2020.

Pre-published: September 10, 2020.

doi:10.3324/haematol.2020.264267

(C)2020 Ferrata Storti Foundation

Material published in Haematologica is covered by copyright. All rights are reserved to the Ferrata Storti Foundation. Use of published material is allowed under the following terms and conditions:

https://creativecommons.org/licenses/by-nc/4.0/legalcode. Copies of published material are allowed for personal or internal use. Sharing published material for non-commercial purposes is subject to the following conditions:

https://creativecommons.org/licenses/by-nc/4.0/legalcode, sect. 3. Reproducing and sharing published material for commercial purposes is not allowed without permission in writing from the publisher. 


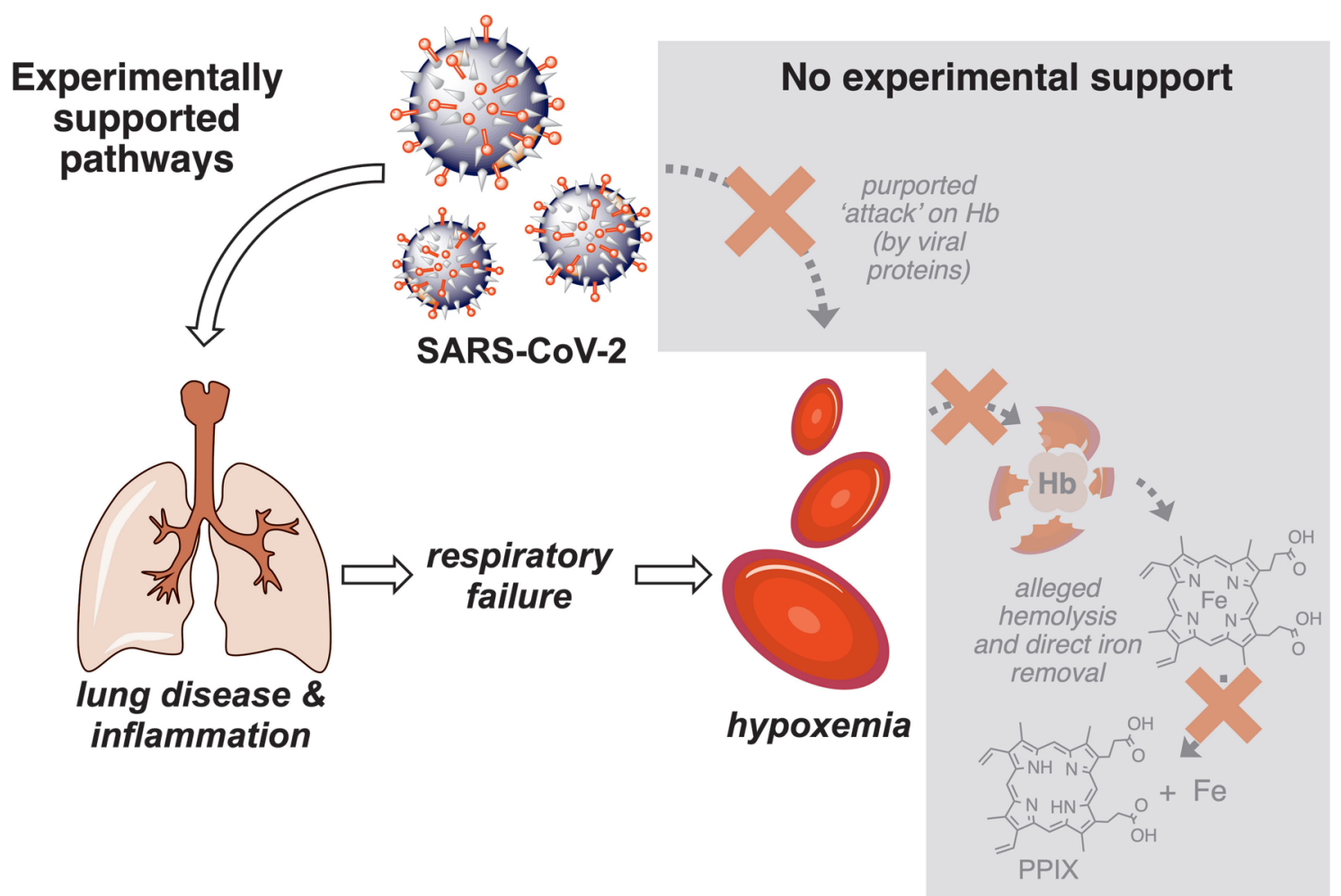

Figure 1. Description of pathophysiology of SARS-CoV-2 infection. Infection via the lungs (open arrows) resulting in hypoxemia (left) vs. direct 'attack' by COVID-19 viral proteins on red blood cell (RBC) hemoglobin $(\mathrm{Hb})$ as proposed by Liu and Li ${ }^{13}$ (right, hashed arrows/gray boxes). Hypothesized pathways crossed out by red X's and highlighted in gray boxes are not supported by available clinical evidence, in vitro/in vivo studies, our results, nor mechanisms of viral interaction with RBC. See text for details.

have reported mortality rates for COVID-19 in the range of $25-32 \%$, similar to the mortality rates for non-COVID19 ARDS. ${ }^{9,10}$ While the exact pathophysiology of COVID19 remains a topic of active investigation, growing evidence suggests that respiratory failure in COVID-19 patients behaves similarly to respiratory failure in patients with severe viral pneumonia that triggers ARDS.11,12 The uncertainty at the outset of this pandemic as well as some persistently unique features of the disease (e.g., increased thromboembolic risks), have led to a large number of proposed hypotheses regarding the pathophysiological mechanisms of SARS-CoV-2.

One widely proposed theory holds that viral proteins directly interact with human hemoglobin $(\mathrm{Hb})$ and facilitate removal of iron from the protein's heme prosthetic group, resulting in the loss of functional hemoglobin and the toxic accumulation of iron. This theory originated from a manuscript by Liu and $\mathrm{Li}^{13}$ posted in the preprint server ChemRxiv (over 1.89 million manuscript views as of August 2020). This work by Liu and Li, ${ }^{13}$ which has not been peer-reviewed and continues to be cited, ${ }^{14,15}$ uses in silico approaches to model interactions between several viral proteins and hemoglobin. In brief, the manuscript suggests that viral proteins ORF1ab, ORF10, and ORF3a are derived from infected plasma cells and work in concert to remove heme from the $\beta$ chain of hemoglobin, strip iron from heme, and sequester the resulting iron-free protoporphyrin IX (PPIX). The authors speculate that this coordinated "attack" of hemoglobin occurs in the plasma following immune hemolysis, resulting in the release of toxic amounts of iron, diminished functional hemoglobin levels, and disrupted heme metabolism. Finally, Liu and
$\mathrm{Li}^{13}$ further purport that the consequences of such hemoglobin degradation account for some of the irregular clinical characteristics reported early in the pandemic (Figure 1). While this work has received significant attention from scientists, physicians, the press, and the general public, the study advances a theory of viral interaction with hemoglobin that is inconsistent with well-characterized mechanisms of physiological heme degradation, ${ }^{16}$ and, critically, advances a pathophysiological mechanism inconsistent with the clinical presentation of COVID-19 patients. Herein, we compare clinical laboratory data from confirmed COVID-19 patients admitted to the intensive care unit (ICU) at University of Pittsburgh Medical Center (UPMC) to patients with ARDS but without COVID-19. These empirical data challenge the theory that SARS CoV2 proteins directly remove iron from human hemoglobin as a pathophysiological mechanism of COVID-19.

\section{Methods}

We reviewed laboratory data from 21 patients with known COVID-19 (PCR positive for SARS-CoV-2) admitted to the ICU at UPMC and compared with 21 patients with non-COVID-19 ARDS from different etiologies, who had been enrolled in the Acute Lung Injury Registry (ALIR) and Biospecimen Repository at UPMC. Patient data were de-identified. This study was approved by the University of Pittsburgh Institutional Reviewer Board.

We recorded clinically available venous blood gas values of partial pressure of carbon dioxide ( $\mathrm{PvCO} 2$ ), partial pressure of oxygen ( $\mathrm{PvO} 2), \mathrm{pH}$, and venous oxygen saturation of hemoglobin (SvO2). We recorded initial hemoglobin and total bilirubin levels. Values 


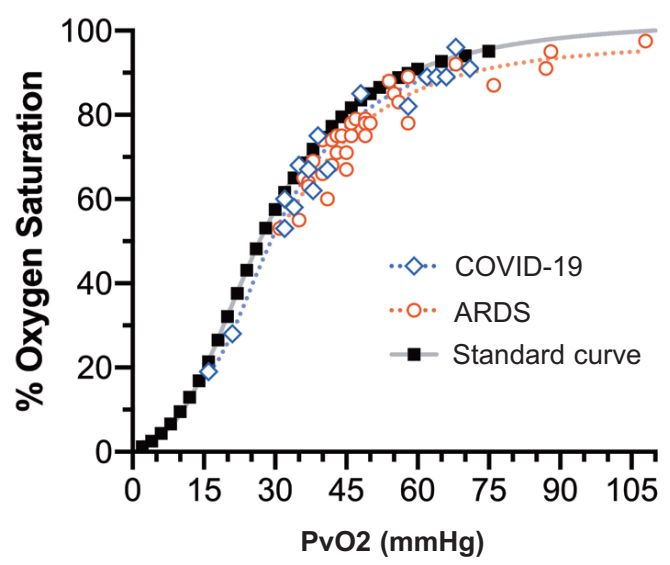

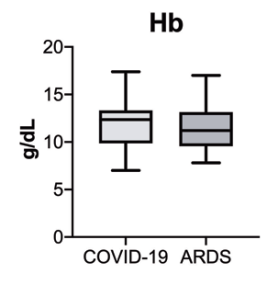
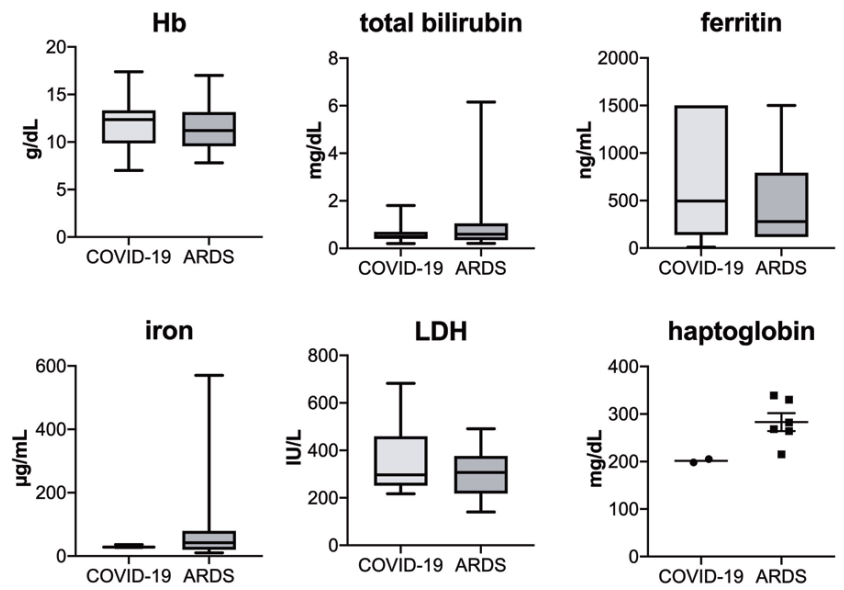

Figure 2. Summary of clinical data of COVID-19 and non-COVID-19 acute respiratory distress syndrome patient cohorts. Oxygen-hemoglobin dissociation curve (left): standard curve as described by JW Severinghaus ${ }^{17}$ (black squares, gray line), values of oxygenation saturation plotted versus PvO2 for patients admitted to intensive care unit (ICU) with COVID-19 (blue diamonds) and ICU patients with acute respiratory distress syndrome (ARDS) but without COVID-19 (red circles). Individual replicates (i.e., same Pv02) are displayed. Corresponding laboratory values of hemoglobin, total bilirubin, ferritin, iron, and lactate dehydrogenase (LDH) were similar between ICU patients with COVID-19 and those with ARDS without COVID-19 (right, no significant differences: $P=0.76, P=0.28, P=0.82, P=0.52, P=0.94$, respectively). Few patients had haptoglobin data, but available COVID-19 values were similar to ARDS (mean 202 vs. 283, respectively). Error bars represent standard error of mean.

for iron, ferritin, haptoglobin, and lactate dehydrogenase (LDH) levels (whenever available during the inpatient admission) were collected as well.

Hemoglobin and total bilirubin levels between COVID-19 and non-COVID-19 patients were compared using a Student's t-test. For iron, ferritin and LDH, Mann-Whitney testing was used. Values for $\mathrm{SvO} 2$ versus $\mathrm{PvO} 2$ were plotted to determine a line of best fit. The deviation of the PvO2 by COVID-19 and ARDS cohorts from the theoretical-standard-predicted-PvO2 (described by JW Severinghaus ${ }^{17}$ ) was evaluated using a Pearson correlation and a coefficient of variation of the root-mean-square deviation or CV(RMSD). All data variance is described as standard error of mean, except for age, which is described as standard deviation.

\section{Results}

Oxygen-hemoglobin dissociation curves were generated using COVID-19 patient data and non-COVID-19 ARDS patient data and compared against the theoretical standard curve generated by the Severinghaus model. ${ }^{17}$ The average age of the COVID-19 cohort was $62 \pm 9$ years versus $47 \pm 17$ years for the ARDS cohort. The distribution of sex was 12 males and 9 females in the COVID-19 cohort and 10 males and 11 females in the non-COVID-19 ARDS cohort. The oxygen-hemoglobin dissociation curve generated from patients with COVID-19 was similar to the curve generated from patients with ARDS without COVID-19. The fitting generated from COVID-19 patient data matched the ideal oxygen-hemoglobin dissociation curve well (Pearson correlation $\mathrm{R}^{2}=0.97, \mathrm{P}<0.0001$; $\mathrm{CV}(\mathrm{RMSD})=7.3 \%)$. The fitting generated from nonCOVID-19 ARDS patients also matches the ideal oxygenhemoglobin dissociation curve reasonably well (Pearson correlation $\mathrm{R}^{2}=0.92, P<0.0001 ; \mathrm{CV}(\mathrm{RMSD})=9.4 \%$, Figure 2). This comparison of oxygen-hemoglobin dissociation curves suggests that hemoglobin oxygen affinity is not altered in patients with COVID-19 admitted to the ICU.

Patients with COVID-19 had similar total hemoglobin, total bilirubin, ferritin, iron and LDH levels compared to patients with ARDS without COVID-19 (none were significantly different, Figure 2). Few patients had haptoglobin levels available. In those COVID-19 patients that did, the average haptoglobin level was similar to that of ARDS patients (mean $205 \mathrm{mg} / \mathrm{dL}, \mathrm{n}=2$, vs. $283 \pm 19$, $\mathrm{n}=6$ ). There was no laboratory evidence of on-going red blood cell hemolysis or degradation of hemoglobin.

\section{Discussion}

Liu and $\mathrm{Li}^{13}$ generated computational results obtained by sequence analysis, molecular modeling, and docking approaches to propose a novel model of viral degradation of hemoglobin-derived heme. However, the authors employed methodologies and docking simulations that have been heavily criticized, as thoroughly discussed in a recent report by Read. ${ }^{18}$ It is important to note that Liu and $\mathrm{Li}^{13}$ do not present any experimental support for their theories, and even though they have revised the initial manuscript and vastly changed their calculated parameters (as of version 9), their new calculations are speculative at best, as Read has recently addressed in an addendum to his manuscript (version 2). ${ }^{18}$ Moreover, the hypotheses originally put forth by $\mathrm{Liu}$ and $\mathrm{Li}^{13}$ remain unchanged. Our work thus focuses on the unique mechanism of SARSCoV-2 proposed by Liu and $\mathrm{Li}^{13}$ direct virus triggered hemoglobin degradation. We highlight that this hypothesis is not supported by existing evidence, known pathologies of coronaviruses, Liu and $\mathrm{Li}$ own docking calculations, ${ }^{18}$ or the clinical data presented herein.

Most nonstructural viral proteins of coronaviruses are not found in large amounts in plasma but rather localize in infected cells where they play important roles in RNA replication. ${ }^{19}$ Thus, these proteins are unlikely to access appreciable amounts of hemoglobin. There is no evidence suggesting that the virus enters erythrocytes, where heme concentrations are $15-20 \mathrm{mM}$, and these highly specia- 
lized cells lack the requisite cellular machinery needed to produce viral proteins. ${ }^{20}$ As a result, intraerythrocytic hemoglobin is likely protected from exposure to viral proteins. Liu and $\mathrm{Li}^{13}$ posit that the interaction between viral proteins and hemoglobin may occur in the plasma after immune hemolysis, but significant hemolysis has not been documented in COVID-19 patients, ${ }^{8,21,22}$ nor did we observe hemolysis in our patients. Any elevation in $\mathrm{LDH}$ levels seen in COVID-19 patients is likely derived from hepatocellular injury and not intravascular hemolysis. Liu and $\mathrm{Li}^{13}$ suggest that the virus could infect plasma cells via the ACE2 receptor and induce secretion of viral proteins from infected plasma cells. However, there is no evidence of SARS-CoV-2 infecting plasma cells. ${ }^{23,24}$ Further, secretion of viral proteins from any infected cells is extremely rare and does not occur in any viruses related to SARSCoV-2. ${ }^{25}$

The manuscript's flawed ${ }^{18}$ and experimentally unverified docking models lead the authors to suggest that a non-structural viral protein, ORF10, binds to heme and releases heme-derived iron. In this putative mechanism, ORF1ab and ORF3a bind the hemoglobin protein and cause conformational changes that expose the heme to ORF10, which subsequently breaks down the cofactor into iron and PPIX; the latter is then theoretically captured by ORF1ab. This model would represent an entirely novel mechanism of hemoglobin degradation, as hemoglobin has not been documented to undergo large conformational shifts as a result of protein-protein interactions. ${ }^{26}$ Further, the removal of iron from PPIX by ORF10, a protein of only 38 amino acids, is unlikely considering that heme degradation is catalyzed by significantly larger and more complex, well-characterized heme-oxygenase proteins. ${ }^{16}$ As the authors' hypothesis represents a completely novel and unexpected model of heme degradation, careful in vitro and in vivo studies would be required to confirm such a mechanism. It is worth noting that we do not observe a statistical difference in iron, bilirubin, or ferritin levels between COVID-19 patients and ARDS controlpatients (Figure 2), indicating that heme breakdown is not occurring above typical catabolic levels in the COVID-19 cohort.

We further assert that the clinical syndrome observed in COVID-19 patients is not consistent with Liu and Li's ${ }^{13}$ model of heme degradation. The clinical evidence presented here does neither suggest hemolysis, hemoglobin degradation, removal of iron from the heme molecule, nor altered oxygen affinity of hemoglobin (Figure 2). The oxygen dissociation curve calculated from real-life patient data fits quite well with the idealized standard curve in both the COVID and ARDS cohorts $\left(\mathrm{R}^{2}=0.97, \mathrm{R}^{2}=0.92\right.$, respectively). There is minimal deviation from the idealized curve CV(RMSD) of 7.3 and $9.4 \%$, respectively). This finding is in agreement with another recent study that uses hemoglobin isolated from the red blood cells of COVID-19 patients demonstrating normal hemoglobinoxygen dissociation properties ex vivo. ${ }^{27}$

The clinical data presented here suggests no significant hemolysis or abnormal hemoglobin-oxygen dissociation characteristics. As aforementioned, newer clinical reports of critically ill COVID-19 patients suggest both mortality rates and mechanical ventilation requirements similar to other forms of ARDS.,7-10 There has been no evidence to suggest a unique hemoglobin-specific mechanism such as large-scale hemoglobin degradation. In addition to the work here, there has been no other evidence of significant anemia or iron overload. ${ }^{8,21,22}$ While the finding of elevated transaminase levels has been widely described, this condition exists without hyperbilirubinemia, which would be a signal of excessive hemolysis and release of hemoglobin into the plasma. ${ }^{26,28}$ Increased risk of thromboembolism is observed in COVID-19 patients; ${ }^{5,6}$ however, acute infections are known to increase the risk of thromboembolism ${ }^{29}$ and thromboembolism is not generally driven by a hemoglobin-based toxicity. Neither "silent hypoxia", ${ }^{4}$ nor patients who maintain a normal work of breathing before rapid onset of $\mathrm{ARDS}^{1}$ suggest an undetected hemoglobin-based toxicity. Any removal of iron and/or heme from hemoglobin would not have an effect on pulse oximetry measurements, as the resulting heme-free hemoglobin does not absorb light in the wavelength range used by these detectors and thus would not interfere with such measurements. ${ }^{30,31}$ Hemoglobin desaturation correlates well with decreases in partial pressure of oxygen in patients with COVID-19 respiratory failure: in our cohort, we did not observe gross abnormalities in the partial pressure of oxygen versus hemoglobin oxygen saturation.

The world community is rapidly working to understand the pathophysiology of COVID-19 in an effort to better prevent the spread of the disease, manage patients, and ultimately develop definitive therapies. There is no suggestion that patients with COVID-19 exhibit a hemolytic anemia or a shift in the normal hemoglobin-oxygen dissociation curve. Thus, COVID-19 does not impact oxygen delivery through a mechanism involving red cell hemolysis and removal of iron from the heme prosthetic group in hemoglobin.

\section{Funding}

This work is funded by 2 P01 HL114453-06 to BJM and 1 K08 HL136857 to JJR.

\section{References}

1. Gattinoni L, Chiumello D, Caironi P, et al. COVID-19 pneumonia: different respiratory treatments for different phenotypes? Intensive Care Med. 2020;46(6):1099-1102.

2. Yang X, Yu Y, Xu J, et al. Clinical course and outcomes of critically ill patients with SARS-CoV-2 pneumonia in Wuhan, China: a single-centered, retrospective, observational study. Lancet Respir Med. 2020; 8(5):475-481

3. Bellani G, Laffey JG, Pham $T$, et al.
Epidemiology, patterns of care, and mortality for patients with acute respiratory distress syndrome in intensive care units in 50 countries. JAMA. 2016;315(8):788-800

4. Xie J, Tong Z, Guan X, Du B, Oiu H, Slutsky AS. Critical care crisis and some recommendations during the COVID-19 epidemic in China. Intensive Care Med. 2020;46(5):837-840.

5. Cui S, Chen S, Li X, Liu S, Wang F. Prevalence of venous thromboembolism in patients with severe novel coronavirus pneumonia. J Thromb Haemost. 2020; 18(6):1421-1424.
6. Klok FA, Kruip MJHA, van der Meer NJM, et al. Confirmation of the high cumulative incidence of thrombotic complications in critically ill ICU patients with COVID-19: an updated analysis. Thromb Res. 2020;191:148-150.

7. Bhatraju PK, Ghassemieh BJ, Nichols M, et al. Covid-19 in critically ill patients in the Seattle region - case series. N Engl J Med. 2020;382(21):2012-2022.

8. Guan W, Ni Z, Hu Y, et al. Clinical characteristics of coronavirus disease 2019 in China. N Engl J Med. 2020;382(18):17081720 . 
9. Richardson S, Hirsch JS, Narasimhan M, et al. Presenting characteristics, comorbidities, and outcomes among 5700 patients hospitalized with COVID-19 in the New York City area. JAMA. 2020;323(20):2052-2059.

10. Public Health Scotland Communications, editor. Scottish Intensive Care Society Audit Group report on COVID-19. 13 May 2020.

11. Ziehr DR, Alladina J, Petri CR, et al. Respiratory pathophysiology of mechanically ventilated patients with COVID-19: a cohort study. Am J Respir Crit Care Med. 2020;201(12):1560-1564

12. Bos LD, Paulus F, Vlaar APJ, Beenen LFM, Schultz MJ. Subphenotyping ARDS in COVID-19 patients: consequences for ventilator management. Ann Am Thorac Soc. 2020 Sep;17(9):1161-1163.

13. Liu W, Li H. COVID-19: Attacks the 1-Beta Chain of Hemoglobin and Captures the Porphyrin to Inhibit Human Heme Metabolism. ChemRxiv. 2020 July 7, v9. [Epub ahead of print].

14. Soy M, Keser G, Atagündüz P, Tabak F, Atagündüz I, Kayhan S. Cytokine storm in COVID-19: pathogenesis and overview of anti-inflammatory agents used in treatment. Clin Rheumatol. 2020;39(7):20852094.

15. Baidya A, Singh SK, Bajaj S, et al. Diabetes and COVID-19: A Review. J ASEAN Fed Endocr Soc. 2020:35(1):40-48.

16. Gozzelino R, Jeney V, Soares MP.
Mechanisms of cell protection by heme oxygenase-1. Annu Rev Pharmacol Toxicol. 2010:50:323-354.

17. Severinghaus JW. Simple, accurate equations for human blood $\mathrm{O} 2$ dissociation computations. J Appl Physiol. 1979; 46(3):599-602.

18. Read R. Flawed methods in "COVID-19: attacks the 1-beta chain of hemoglobin and captures the porphyrin to inhibit human heme metabolism." ChemRxiv. 2020 May 5, v2. [Epub ahead of print].

19. Snijder EJ, Decroly E, Ziebuhr J. The nonstructural proteins directing Coronavirus RNA synthesis and processing. Adv Virus Res. 2016;96:59-126.

20. Asher DR, Cerny AM, Finberg RW. The erythrocyte viral trap: transgenic expression of viral receptor on erythrocytes attenuates coxsackievirus B infection. Proc Natl Acad Sci U S A. 2005;102(36):1289712902.

21. Fan BE, Chong VCL, Chan SSW, et al. Hematologic parameters in patients with COVID-19 infection. Am J Hematol. 2020 95(6): e131-134

22. Mitra A, Dwyre DM, Schivo M, et al Leukoerythroblastic reaction in a patient with COVID-19 infection. Am J Hematol. 2020;95(8):999-1000.

23. Thevarajan I, Nguyen THO, Koutsakos M, et al. Breadth of concomitant immune responses prior to patient recovery: a case report of non-severe COVID-19. Nat Med. 2020;26(4):453-455

24. Zhu Y, Jiang M, Gao L, Huang X. Single cell analysis of ACE2 expression reveals the potential targets for 2019-nCoV. Preprints. $2020 \mathrm{Feb}$ 16. [Epub ahead of print].

25. Schatz M, Tong PBV, Beaumelle B Unconventional secretion of viral proteins. Semin Cell Dev Biol. 2018;83:8-11.

26. Stites WE. Protein-protein interactions: interface structure, binding thermodynamics, and mutational analysis. Chem Rev. 1997;97(5):1233-1250.

27. Daniel Y, Hunt BJ, Retter A, et al Haemoglobin oxygen affinity in patients with severe COVID-19 infection. Br Haematol. 2020;190(3):e126-127.

28. Fan Z, Chen L, Li J, et al. Clinical features of COVID-19-related liver functional abnormality. Clin Gastroenterol Hepatol. 2020; 18(7):1561-1566

29. Smeeth L, Cook C, Thomas S, Hall AJ Hubbard R, Vallance P. Risk of deep vein thrombosis and pulmonary embolism after acute infection in a community setting. Lancet. 2006;367(9516):1075-1079.

30. Pires IS, Belcher DA, Palmer AF. Quantification of active apohemoglobin heme-binding sites via dicyanohemin incorporation. Biochemistry. 2017; 56(40):5245-5259.

31. Jubran A. Pulse oximetry. Crit Care Lond Engl 2015;19(1):272. 